\title{
Is it important to quantify hepatic fibrosis?
}

\author{
Anirudh Kohli \\ Breach Candy Hospital Trust, 60 Bhulabhai Desai Road, Mumbai, Maharashtra, India. E-mail: dranirudhkohli@gmail.com
}

Metabolic syndrome represents a cluster of conditions that occur together, increasing the risk of developing heart disease, stroke, and type II diabetes. Metabolic syndrome causes a 2-4 fold increase in stroke, 3-4 fold increase in myocardial infarction, and a 5 fold increase in developing diabetes. It is estimated that $25 \%$ of world population suffers from metabolic syndrome. In India studies have shown incidence as high as $41 \%{ }^{[1]}$

Urbanization, high calorie diet, lack of physical activity, and obesity are important factors in developing metabolic syndrome.

The hepatic manifestation of metabolic syndrome is hepatic steatosis. Insulin resistance in metabolic syndrome results in cells not responding normally to insulin, resulting in excessive fat deposition. The presence of hepatic steatosis has an important impact on the health of an individual. Over $20-50 \%$ of individuals with hepatic steatosis will become diabetic. Hepatic steatosis increases the risk of mortality by $34 \%$ compared to the general population. ${ }^{[2]}$

Sonography is the most common modality to detect hepatic steatosis. The presence and severity is based on qualitative sonographic features of echogenicity, echotexture, vessel visibility, and beam attenuation. However, quantitative estimation of hepatic steatosis is not possible. CT scanning also detects hepatic steatosis qualitatively but quantification is not possible. MRI is the most sensitive modality to detect and quantity fat.

Chemical shift imaging utilizes in and out phase images to exploit the difference between resonance frequencies of water and fat signals. In the in phase, their resonance frequencies is aligned in the same direction, and thus their signals add up. In the out phase, their signals cancel each other, thus the quantum of fat can be estimated.

MR spectroscopy utilizes the principle that water and fat have different resonant frequencies. MR spectroscopy is the most accurate modality to detect quantum of fat. Unfortunately it only samples a small portion of liver and does not provide a global overview which chemical shift imaging does. Normal liver fat percentage is $5 \% .{ }^{[2]}$
The main hepatic complication of hepatic steatosis is the development of steatohepatitis, which may progress to fibrosis and consequently cirrhosis. There is an increased risk of developing hepatocellular carcinoma once cirrhosis develops. Hepatic fibrosis has been considered an irreversible process, but now is considered to be a dynamic process with potential for regression. Therefore, it is important to develop a technique to detect hepatic fibrosis. It is also important to quantify hepatic fibrosis to monitor disease progression as well as response to conventional and newer drugs.

Liver biopsy is the gold standard for demonstrating liver fibrosis as well as grading/staging liver fibrosis. Unfortunately it has its share of drawbacks: it is invasive with possible complications of hemorrhage and a small but documented fatality rate. Only a small area of liver is sampled, underestimating the extent and degree of fibrosis. This has been borne out by comparative studies between liver biopsy and laproscopy. In addition, the histological evaluation is subjective varying from histopathologist to histopathologist. As fibrosis is a dynamic process, assessment of fibrosis is required repeatedly during the course of treatment and illness. Performing repeated liver biopsy is not possible. It is therefore important to have a technique which is noninvasive, free of complications, samples the entire liver, and removes subjectivity.

Due to the limitations of liver biopsy, noninvasive techniques have been introduced to detect liver fibrosis. Elastography is an imaging technique that evaluates the stiffness of tissue based on the velocity of propagation of mechanical waves. Mechanical waves travel faster in stiffer tissues and slower in soft tissues. These techniques detect liver stiffness, termed as liver stiffness measurements (LSM). In these methods an external acoustic force is applied to the liver. This force temporarily distorts hepatic parenchyma producing shear waves that travel through the liver perpendicular to the direction of the force. This sheer wave velocity is related to tissue stiffness. Shear waves travel faster in stiff tissues and slower in soft tissues. The velocity of these shear waves can be identified by sonography or MR helping determine the extent of fibrosis. 


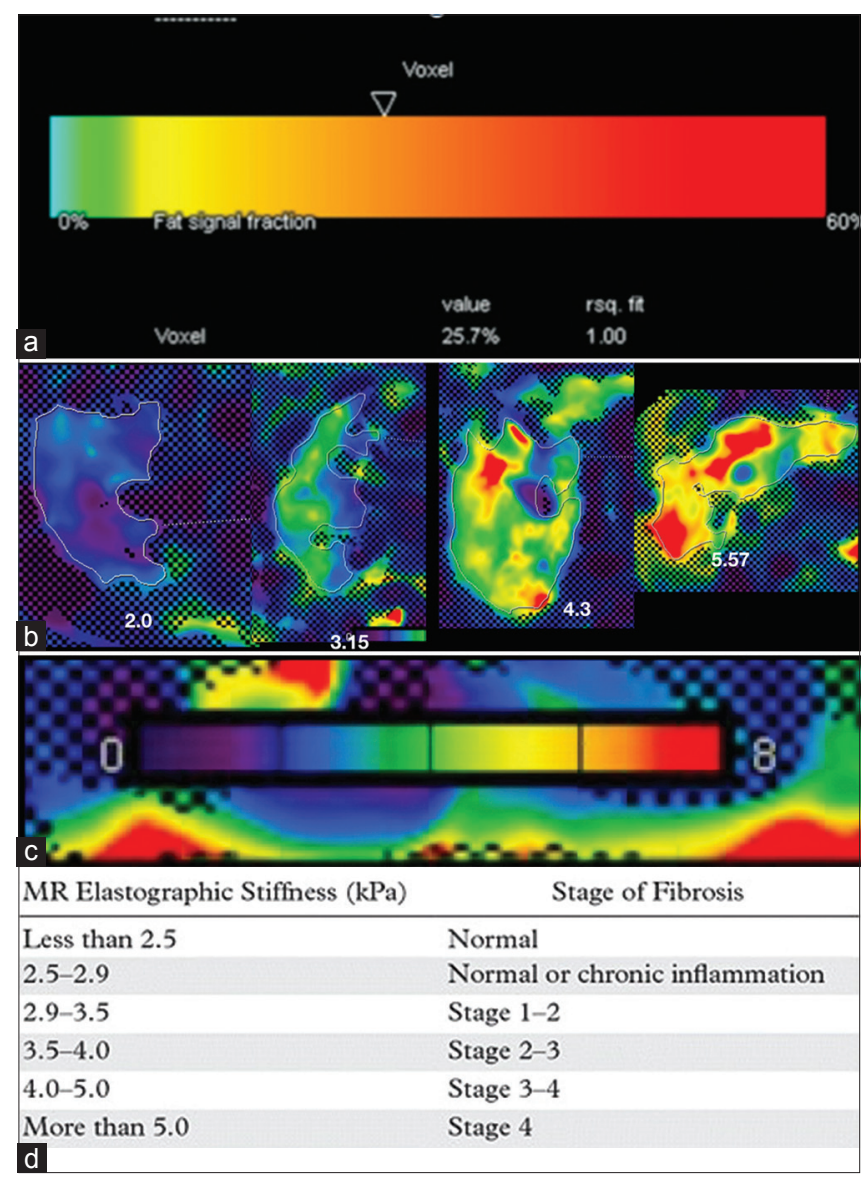

(a) Fat signal fraction: Chemical shift imaging Quantifying fat percentage $(25.7 \%)$. (b) MR elastography quantification of fibrosis: Four cases demonstrating range of fibrosis from 2.09, 3.15, 4.3, and 5.5. (c) MR elastography color scale of stiffness from 0-8 Kpa. (d) MR Elastography relationship between Kpa range and stage of fibrosis

MR elastography is the most accurate of all the techniques in terms of identification and staging of fibrosis. It has advantages over sonography as it is more accurate and evaluates the entire liver compared to sonography which evaluates only small portions of the Liver.
Detection of hepatic steatosis is important not only because it can progress to fibrosis but also because hepatic steatosis may bring attention to the possibility of metabolic syndrome, which in turn may bring attention to the possibility of cardiovascular, cerebrovascular disease, and diabetes. Thus, helping reduce the adverse events consequent to these diseases. A useful starting point is a liver sonography, if there is hepatic steatosis, quantify using chemical shift MRI and check and quantify fibrosis with MR elastography. To follow up hepatic steatosis chemical shift MRI is adequate, to follow up hepatic fibrosis MR elastography is essential.

\section{References}

1. Khan Y, Lalchandani A, Gupta AC, Khadanga S, Kumar S. Prevalence of metabolic syndrome crossing $40 \%$ in Northern India: Time to act fast before it runs out of proportions. J Family Med Prim Care 2018;7:118-23.

2. Benedict $M$, Zhang $X$. Non-alcoholic fatty liver disease: An expanded review. World J Hepatol 2017;9:715-32.

This is an open access journal, and articles are distributed under the terms of the Creative Commons Attribution-NonCommercial-ShareAlike 4.0 License, which allows others to remix, tweak, and build upon the work non-commercially, as long as appropriate credit is given and the new creations are licensed under the identical terms.

\begin{tabular}{|l|l|}
\hline \multicolumn{2}{|c|}{ Access this article online } \\
\hline Quick Response Code: & Website: \\
& www.ijri.org \\
\hline & \\
& \\
\hline
\end{tabular}

Cite this article as: Kohli $A$. Is it important to quantify hepatic fibrosis? Indian J Radiol Imaging 2019;29:341-2.

Received: 27-Dec-2019

Accepted: 27-Dec-2019 Published: 31-Dec-2019 